\title{
Prevalencia de hendidura labiopalatina
}

\section{Prevalence of labiopalatine hending}

\section{Prevalencia de hendidura labiopalatina}

\author{
Rosangelina Lucena \\ rosangelina18@gmail.com \\ Kasandra Uzcátegui \\ kasandra.uzcategui@gmail.com
}

Recibido mayo 2019 / Revisión junio 2019 / Aceptado 1 de septiembre 2019

\begin{abstract}
RESUMEN
Introducción: La hendidura labio-palatina es un defecto congénito, donde existe la separación del labio superior y fisura del maxilar, por la fusión incompleta de los procesos maxilares y naso-medial del embrión, que conduce a múltiples alteraciones. Objetivo: Determinar la prevalencia de hendidura labio-palatina en pacientes que acuden a la Asociación Valenciana, para la atención interdisciplinaria de pacientes con alteraciones cráneo-faciales, Valencia-Carabobo/Venezuela, en el período 2016-2017. Materiales y métodos: Se realizó una investigación de campo, descriptiva, con el apoyo de historias clínicas. Con una población de 93 pacientes y una muestra pertinente de 45 pacientes; plasmando la información en gráficos y cuadros por frecuencia y \% simple. Resultados: La hendidura labio-palatina bilateral, se presenta con mayor frecuencia que la unilateral, puede ser derecha e izquierda; todas predominantes en el sexo masculino y en edades de 0 - a 9 años. Conclusión: Esta alteración acarrea problemas estéticos, alimenticios, auditivos, respiratorios y foniátricos, que deben ser abordados por un grupo multidisciplinario. Cada vez su prevalencia es alta, lo que conlleva a crear instituciones especializadas, con carácter resolutivo, para dar respuestas rápidas, a las diferentes malformaciones cráneo-faciales.
\end{abstract}

Palabras clave: prevalencia; hendidura; palatina; malformación
RL: Universidad José Antonio Páez, Venezuela.

KU: Universidad José Antonio Páez, Venezuela.

\begin{abstract}
Introduction: The cleft lip-palate is a congenital defect, where there is a separation of the upper lip and fissure of the maxilla, due to the incomplete fusion of the maxillary and naso-medial processes of the embryo, which leads to multiple alterations. Objective: To determine the prevalence of cleft palate in patients who come to the Valencian Association, for the interdisciplinary care of patients with craniofacial disorders, Valencia-Carabobo / Venezuela, in the period 2016-2017. Materials and methods: A descriptive field research was carried out, supported by medical records. With a population of 93 patients and a relevant sample of 45 patients; expressing the information in graphs and charts by frequency and simple\%. Results: The bilateral cleft lip-palate, occurs more frequently than the unilateral, can be right and left; all predominant in the male sex and in ages 0-9 years. Conclusion: This alteration leads to aesthetic, nutritional, auditory, respiratory and phonatal problems, which must be addressed by a multidisciplinary group. Each time its prevalence is high, which leads to creating specialized institutions, decisively, to give quick responses to the different craniofacial malformations.
\end{abstract}

Key words: prevalence; cleft palatine; malformation 
RL: Universidad José Antonio Páez, Venezuela.

KU: Universidad José Antonio Páez, Venezuela.

\section{RESUMO}

Introdução: A fissura labiopalatina é um defeito congênito, onde há separação do lábio superior e fissura da maxila, devido à fusão incompleta dos processos maxilar e nasomedial do embrião, o que leva a múltiplas alterações. Objetivo: Determinar a prevalência de fissura palatina em pacientes que ingressam na Associação Valenciana, para o atendimento interdisciplinar de pacientes com distúrbios craniofaciais, Valencia-Carabobo / Venezuela, no período 20162017. Materiais e métodos: Foi realizada uma pesquisa de campo descritiva, apoiada em prontuários médicos. Com uma população de 93 pacientes e uma amostra relevante de 45 pacientes; expressar as informações em gráficos e tabelas por frequência e\% simples. Resultados: A fissura labial palatina bilateral, ocorre com mais frequência que a unilateral, pode ser direita e esquerda; todos predominam no sexo masculino e na faixa etária de 0 a 9 anos. Conclusão: Essa alteração leva a problemas estéticos, nutricionais, auditivos, respiratórios e fonológicos, os quais devem ser abordados por um grupo multidisciplinar. Cada vez que sua prevalência é alta, o que leva à criação de instituições especializadas, decisivamente, para dar respostas rápidas às diferentes malformações craniofaciais.

Palavras-chave: prevalência; hendidura; palatina; malformação

\section{INTRODUCCIÓN}

a hendidura labio-palatina es un $\mathrm{L}$ defecto congénito, que consiste en la separación del labio superior y fisura o grieta del maxilar, por la fusión incompleta de los procesos maxilar y naso-medial del embrión, que produce una comunicación de la boca con la cavidad nasal, la cual puede ser causada por factores genéticos 0 hereditarios; siendo estos uno de los defectos del nacimiento más frecuentes y constituye aproximadamente el 15\% de las malformaciones congénitas.

Cada 500 a 700 recién nacidos, presentan defectos congénitos, como paladar hendido. Esta proporción varía considerablemente dependiendo del grupo étnico y de la zona geográfica (1). La Universidad Complutense de Madrid, resalta que la hendidura labio-palatina es una malformación facial muy frecuente, con una incidencia de $1 / 1100$, debido a la falta de fusión de los procesos maxilares, siendo más frecuente en el sexo masculino, afectación unilateral derecha; mientras que su asociación con paladar hendido es de 1/2500 nacimientos, más en el sexo femenino y en hijos de padres con edad avanzada (2).

El Ministerio de Salud y Protección Social Colombia (3), afirma que la prevalencia de labio y paladar hendido asociados es de $0.07 \%$ en el país, mientras el reporte de estos eventos se da en menor proporción si se hace referencia a fisura labial o paladar hendidura labial para Bogotá, es de $0.27 \%$.

En relación con la distribución por sexo, se encuentra la fisura en el $0.3 \%$ en varones y $0.17 \%$ en mujeres, siendo el $91.68 \%$ de las fisuras labiales bilaterales. Mientras, que en Venezuela (4) para el año 2005 se encontró en una investigación que la prevalencia de hendidura labial y/o palatina en pacientes neonatos es del $12 \%$, en una población de 481 niños con hendidura labial y/o palatina.

En relación a su ubicación geográfica, el municipio Valencia presentó $76 \%$ y Guacara y Bejuma 13\%; con un $75 \%$ de padres con edad que no sobrepasan los 34 años al momento de la procreación (4). De allí, la importancia del manejo de la prevalencia de la malformación congénita hendidura labio-palatina, ya que permitirá 
a los especialistas del área de cirugía bucal y maxilofacial precisar la frecuencia con la cual se presenta este defecto facial y así poner en práctica medidas para la solución de dicha afectación.

En Ecuador se realizó una investigación sobre la Prevalencia de Fisura Labio-Palatina en niños donde se detectó que la fisura labio-palatina es la más común; unilateral derecha en menores de 3 años, con un 27\% (5). En Guatemala en un estudio sobre la caracterización de pacientes con labio y paladar hendido y se evidenció $64.81 \%$ en relación al sexo femenino $35.19 \%$. El tipo de malformación predominante fue el labio hendido unilateral completo con afectación del paladar (6). Los investigadores determinaron el predominio de pacientes con labio y/o paladar hendido del sexo masculino. El tipo de malformación más frecuente dentro de los casos es el labio hendido unilateral completo, con afectación del paladar.

En Chiclayo - Perú, (7) se llevó a cabo un trabajo sobre la frecuencia de neonatos con fisura del paladar y labio leporino en dos Hospitales de la Región Lambayeque el cual arrojó que la mayor frecuencia de la malformación fue la fisura del paladar y labio leporino con 4 casos $(1,5 \%)$ y labio leporino con 1 caso $(0,4 \%)$ haciendo un total de 5 casos con malformación (1,9\%) de la población total. Es importante la realización de estadísticas periódicas, en los diferentes hospitales, donde nacen neonatos con malformaciones, lo cual permitiría la disposición de una información actualizada de estas patologías; favoreciendo su prevención y tratamiento.

En el estado Mérida en Venezuela (8) se investigó sobre las características epidemiológicas en pacientes pediátricos con hendiduras de labio y paladar entre los años 2006 y 2013, en el Instituto Autónomo Hospital Universitario de Los
Andes (IAHULA), se evidenció que, del total de las historias clínicas evaluadas, el $65.2 \%$ de los niños con HLP eran del sexo masculino y el $60.2 \%$ procedía del área rural, sin antecedentes familiares de dicha patología. Por otro lado, en Facultad de Odontología de la Universidad Central de Venezuela (9). Se realizó un trabajo titulado: Prevalencia de Hendiduras de labio y/o palatinas en los pacientes que acudieron al Centro de Investigación y Atención a Pacientes con Malformaciones Craneo-faciales y Prótesis Maxilofacial durante los años 2000-2012, en el que se reportó una población de 329 niño/as, el $51,1 \%$ varones y el $48,9 \%$ niñas, con edades de 0-5 años 89,1\% en su mayoría, predominando en un 21,3\% HLP completa, $17,3 \%$ unilateral completa izquierda $\mathrm{y}$ $15,5 \%$ derecha. La hendidura que predomino en esta muestra fue de HLP bilateral completa.

Venezuela es un país con una tasa baja de malformaciones congénitas, según estudios realizados tanto en las áreas urbanas y rurales del Distrito Federal y los estados Zulia y Mérida. En el estado Carabobo la prevalencia de hendidura labial y/o palatina es del 12\%. En relación a ubicación geográfica, el Municipio Valencia presentó 76\% y Guacara y Bejuma 13\%; $75 \%$ de los padres no sobrepasan los 34 años al momento de procrear. En cuanto al nivel educativo, $67 \%$ no culmina estudios secundarios. La prevalencia de la hendidura labial y/o palatina es muy alta en el Estado Carabobo. Progenitores, presentaron un nivel educativo bajo y una edad de procrear por debajo de 34 años, es una población joven. Se considera como un problema poli génico multifactorial, influido por factores ambientales, que interactúan con el proceso de morfogénesis de los paladares primario $\mathrm{y}$ secundario, en el que se puede producir hendiduras $(10,11)$. Esta alteración, es 
producida entre la cuarta y doceava semana del embarazo a nivel de las estructuras orofaringonasales y además podrían integrarse a un síndrome más complejo o aparecer de manera aislada $(10,12)$.

La clasificación de las fisuras está dada de acuerdo a las estructuras afectadas ya que se encuentran diversos grados de severidad como: "fisura de labio, fisura labiopalatina y fisura palatina aislada", la categorización de las fisuras se realiza dependiendo de las estructuras comprometidas: labio, encía, paladar óseo, velo (13).

La Hendidura Labial y Palatina acarrea problemas estéticos, alimenticios, auditivos, respiratorios y foniátricos, por lo cual debe ser abordada por un equipo multidisciplinario (10,14-15).

Durante el desarrollo embrionario existen dehiscencias en la unión del paladar primario o secundario llevan a la aparición de fisuras, ya sean del paladar primario (afectan al labio con o sin compromiso del hueso alveolar), fisuras del paladar secundario (afectan paladar óseo y/o blando); mistas (compromiso de labio y paladar) y fisuras atípicas, estas ocurren en menor proporción (16). Asimismo, se pueden ver afectados los huesos del esqueleto, cráneo y cara por el cierre precoz de las suturas craneales denominadas sinostosis, clínicamente se aprecian en caras y cráneos malformadas como consecuencia de un crecimiento anómalo del esqueleto óseo (17).

Las hendiduras pueden clasificarse según su extensión en: a) Unilateral, por fuera de la cresta filtral, todos los elementos de la parte media del labio forman parte del borde interno de la fisura, la cresta filtral de la fisura labial es más corta y más oblicua que la de la mitad opuesta del labio. B) Bilateral, el prelabio, se encuentra separado de las dos porciones laterales, el arco de cupido no es reconocible en este tipo de fisura, puesto que la premaxila se encuentra protruida destruyendo el área de la columela (18).

El objetivo de la presente investigación fue determinar la prevalencia por de hendidura labiopalatina en los pacientes que acuden a la Asociación Valenciana para la Atención Interdisciplinaria de Pacientes con Alteraciones Cráneo-faciales (A.V.A.I.P.A.C.F.), ValenciaCarabobo/Venezuela, en el período 20162017.

\section{MATERIALES Y MÉTODO}

I nvestigación descriptiva y de campo, retrospectiva y transversal, La población estuvo constituida por 93 pacientes que asistieron a la Asociación Valenciana, para la Atención Interdisciplinaria al Paciente con Alteraciones Cráneo-faciales (A.V.A.I.P.A.C.F), de ambos sexos, con edades comprendidas entre 0 y 9 años, de estos 45 pacientes constituyen la muestra a analizar, los datos se tomaron de las historias clínicas y fueron tabulados y analizados, con el uso de estadística descriptiva, con frecuencia y porcentaje simple, y se presentan en cuadros $y$ gráficos.

\section{RESULTADOS Y DISCUSIÓN}

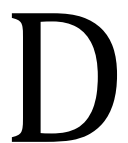

e la población estudiada, un total de 93 pacientes, se analizó el $42 \%$ de las historias, que corresponden a 45 pacientes quienes constituyen la muestra y presentan hendidura labiopalatina, a pesar de ser menos de la mitad del total, hay que tener en cuenta que el resto de las historias clínicas no pertenecen a una sola alteración craneofacial sino a varias (Hendidura palatina, Hendidura labial, Microtía, Atrofia nasal, Síndrome de Moebius, Síndrome de Pierre Robín) por lo tanto es la alteración craneofacial con mayor cantidad de pacientes atendidos según las historias clínicas de la A.V.A.I.P.A.C.F, de 
este total un $51,61 \%$ son pacientes con alteración craneofacial el restante $48,38 \%$ se corresponde con los pacientes que presentan hendidura labiopalatina.

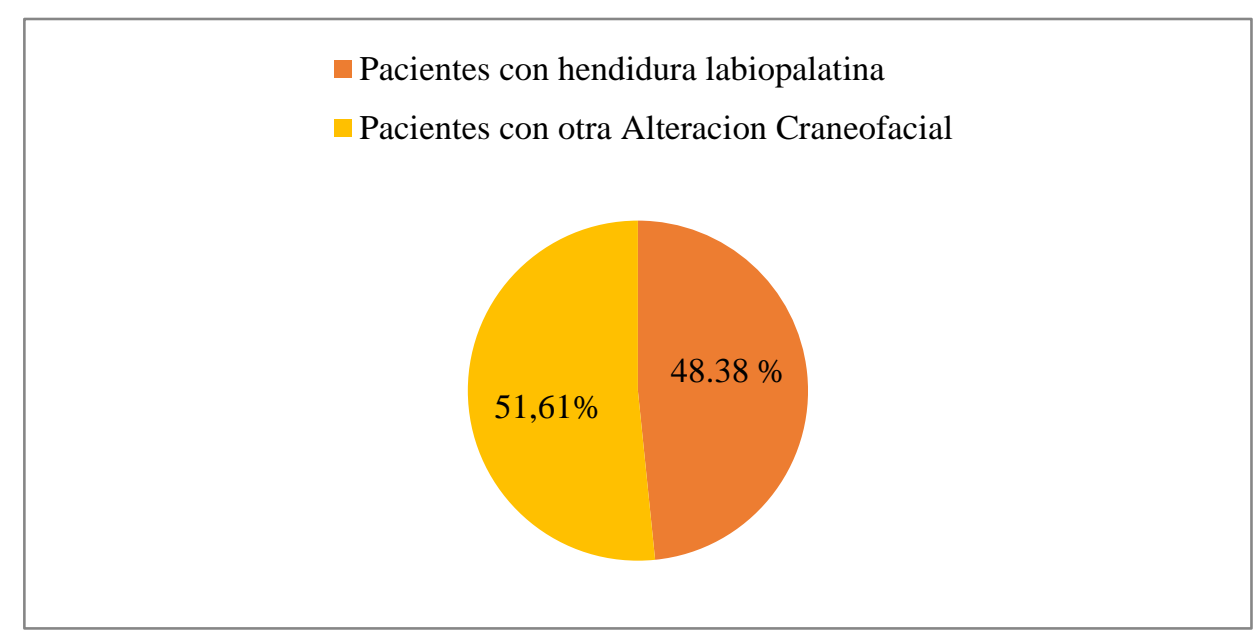

Gráfico 1. Distribución por número de pacientes atendidos A.V.A.I.P.A.C.F. Valencia.2016-2017

(Fuente: datos de la investigación)

En el gráfico 2 se evidencia que el sexo con mayor prevalencia de hendidura labiopalatina es el masculino. Las hendiduras labiopalatinas ya sean bilaterales completas o unilaterales completas, prevalecen en el sexo masculino de manera mayoritaria (gráfico $3)$ así lo reflejan estudios realizados en otros países $(8,9)$

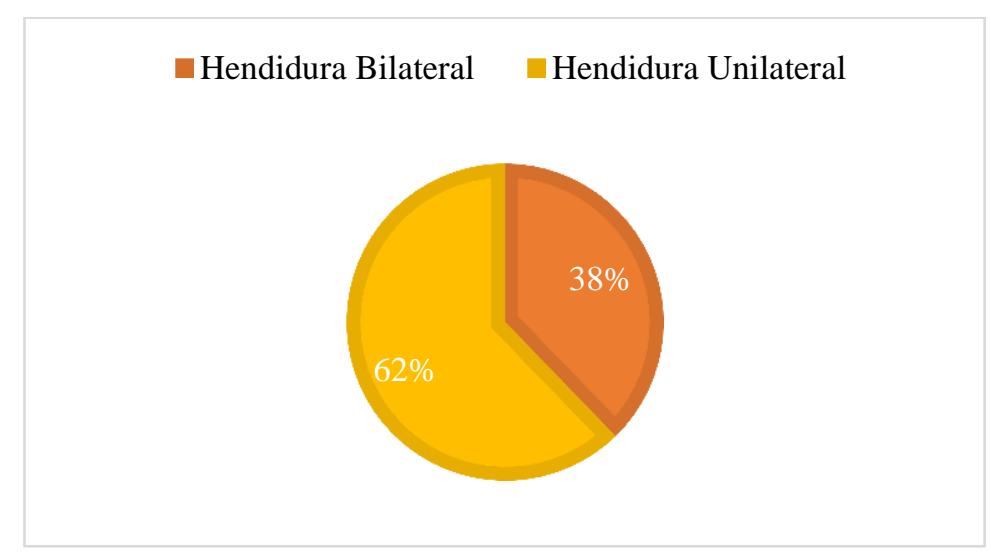

Gráfico 2. Distribución por sexo, en los pacientes que acuden a la A.V.A.I.P.A.C.F. Valencia.20162017. (Fuente: datos de la investigación) 


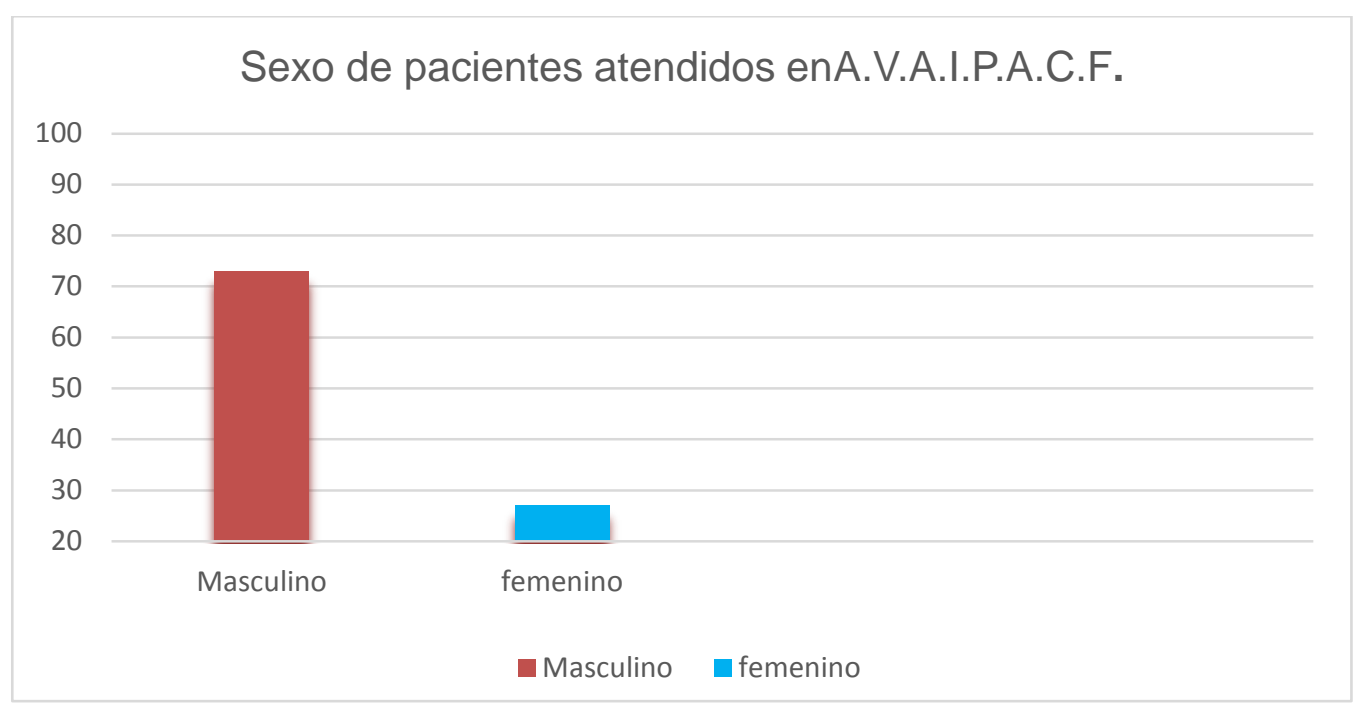

Gráfico 3. Distribución según el tipo de hendidura labiopalatina, presente en los pacientes que acuden a A.V.A.I.P.A.C.F. Valencia.2016-2017. (Fuente: datos de la investigación).

De acuerdo a la clasificación de la hendidura labiopalatina, se observó que 38\% de los pacientes la padecen de forma bilateral, mientras que $62 \%$ de los pacientes presenta la hendidura unilateral. Se observa que la Hendidura Unilateral completa (afecta labio, premaxila y paladar, y puede ser del lado derecho o izquierdo) tiene mayor evidencia que la Hendidura Bilateral, donde se encuentra que el tipo de malformación predominante fue el labio hendido unilateral completo con afectación del paladar.

\section{Tipo de Hendidura Labio-palatina}

70

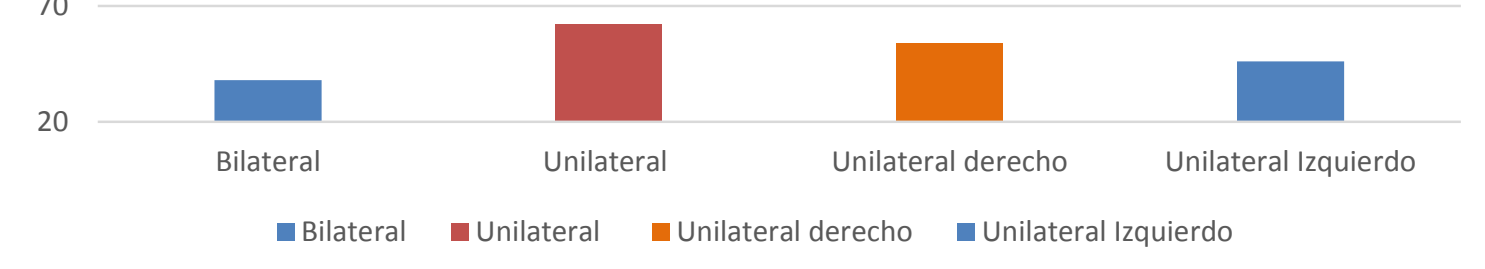

Gráfico 4. Distribución por tipo de hendidura labiopalatina unilateral, en pacientes que acuden a la A.V.A.I.P.A.C.F. Valencia.2016-2017(Fuente: datos de la investigación).

De acuerdo a la clasificación de la hendidura labiopalatina, se observó que $38 \%$ pacientes padecen de hendidura labiopalatina bilateral, mientras que $62 \%$ pacientes la presentan unilateral, de los cuales $54 \%$ corresponden al lado derecho y el restante 46 $\%$ presentan del lado izquierdo. Existen diferentes expresiones clínicas de las fisuras; dependiendo de las estructuras anatómicas que implican y la severidad de estas. Es muy importante caracterizar adecuadamente cada caso, ya que esto determina su diagnóstico, ofrece una guía para su tratamiento y su posible pronóstico. 
La hendidura palatina es la malformación congénita orofacial más frecuente, con una falta de unión de los planos cutáneo, muscular y óseo a nivel del labio superior, fosas nasales, premaxila y apófisis horizontal del maxilar (19). Su etiología es de origen multifactorial con un amplio número de factores contribuyentes, tanto genéticos como ambientales, entre los cuales cabe mencionar la exposición a agentes químicos, la radiación, la hipoxia maternal $o$ restricciones debidas a compromiso del cordón umbilical, la ingesta de fármacos, las deficiencias nutricionales, las obstrucciones físicas y la influencia genética (20).

De acuerdo con Cherfên et al (21), Quijano et al (22) y Chavarriaga et al (23), la incidencia media de hendiduras de labio y/o paladar en la población caucásica es de aproximadamente 1:500 nacidos vivos (nv) en Europa y de 1:700-1000 nv en Estados Unidos, mientras que en la población de origen negro es inferior (0.41: $1000 \mathrm{nv}$ o 1:2500 a $3000 \mathrm{nv}$ ). Señalan también que, en Chile, la incidencia es de 1 por cada $580 \mathrm{nv}$, en Colombia de 1:1000 nv y en Bolivia de 25:10000 nv. Las tasas más altas se presentaron en Bolivia con 23.7 seguidas de Ecuador con14.96 y Paraguay con 13.3 $\mathrm{y}$, por otra parte, las tasas más ajas se presentaron en países como Venezuela con 7.92 (23).

La hendidura palatina se caracteriza por síntomas que afectan a los mecanismos respiratorios, deglutorios, articulatorios, del lenguaje, la audición y de la voz, y repercute marcadamente a nivel estético, sino también en el plano de las relaciones humanas. Su relevancia radica en los elevados costos asociados a su reparación quirúrgica, así como en el impacto psicológico y social del niño que la presenta (24). Por tanto, resulta conveniente identificar todas las condiciones potencialmente asociadas a su aparición con lo cual se hace necesario adicionar y actualizar la información relacionada; así como, impulsar un mayor número de estudios en esta área que contribuyan a comprender mejor el desarrollo de esta patología.

En la presente investigación se encontró que los pacientes que acuden a la A.V.A.I.P.A.C.F, diagnosticados con hendidura palatina pertenecen en su mayoría al sexo masculino, lo cual coincide con lo reportado por otros investigadores que han establecido que los varones están en mayor riesgo en comparación con las hembras $(25,26)$. Se han confirmado estos resultados estadísticos a nivel mundial, cuanto mayor es el defecto, mayores la proporción en los varones (25).De manera que es destacar los aportes de Mossey (27) quien señala que la hendidura labial, con o sin hendidura del paladar, es más frecuente en el sexo masculino, mientras que la fisura del paladar aislada se ha observado más frecuentemente en mujeres, a través de varios grupos étnicos, la proporción de acuerdo al sexo varia con la severidad de la fisura, presencia de malformaciones adicionales, número de hermanos afectados en la familia, etnias, origen y posiblemente la edad paterna.

Las clasificaciones son una forma de organizar la información, de tal forma que sea más comprensible y clara; permitiendo que se desarrolle una comunicación efectiva entre los distintos profesionales de la salud. En la presente investigación se observó la hendidura labio palatina clasificada como unilateral se presentó en $62 \%$ de los pacientes mientras que la bilateral en 38\%. Coinciden estos resultados con la investigación de MenaOlalde (20) realizada en México donde revisaron los casos de 800 pacientes encontrando predominio de la forma unilateral. No obstante, el presente estudio difiere de la investigación de Mena-Olalde (20) donde hubo mayor porcentaje de 
casos del lado izquierdo, en los pacientes de la A.V.A.I.P.A.C.F se encontró predominio del lado derecho. Aproximadamente el $85 \%$ de las fisuras bilaterales y el $70 \%$ de las fisuras labiales unilaterales, están asociadas con fisuras palatinas (25).

\section{CONCLUSIÓN}

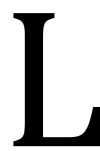
a investigación realizada da cuenta que los pacientes que acuden a la A.V.A.I.P.A.C.F presentaron prevalencia de hendidura labio-palatina coincidente con los resultados arrojados por la literatura internacional consultada. Es la alteración cráneo-facial con mayor cantidad de pacientes atendidos según las historias clínicas revisadas, donde el total de 51,61\% presentó alteración cráneofacial y el restante $48,38 \%$ se correspondió con los que presentaron hendidura labiopalatina.

La persona con fisura labio palatina presenta diversas repercusiones $y$ trastornos que surgen de los cambios anatómicos, funcionales y estéticos; alimenticios, auditivos, respiratorios y foniátricos, que comprometen la vida social del portador quien requerirá varios procedimientos quirúrgicos y complejos tratamientos médicos que deben ser abordados por un grupo multidisciplinario. Su prevalencia cada vez es más alta, lo que conlleva a crear instituciones especializadas, con carácter resolutivo, para dar respuesta a las múltiples malformaciones cráneo-faciales. De allí la necesidad de profundizar en el conocimiento y tratamiento de esta patología.

- Los autores declaran no poseer conflictos de interés

- Estudio realizado bajo los estatutos bioéticos

- Ente financiador: La investigación no conto con financiamiento alguno

\section{REFERENCIAS BIBLIOGRÁFICAS}

1.- Organización Mundial de la Salud. Salud bucodental. [Internet] Nota informativa $\mathrm{N}^{\circ} 318,2012$. [Citado 12 agosto 2017]. Disponible en: http://www.who.int/mediacentre/fac tsheets/fs318/es/

2.- Universidad Complutense de Madrid. Anomalías Faciales Congénitas. [Internet] UCM Madrid.2012. [Citado 14 agosto 2017]. Disponible en: https://www.ucm.es/data/cont/docs /420-2014-02-26-

08\%20Anomalias\%20congenitas.pdf

3.- Ministerio de Salud y Protección Social. Colombia. Cuarto Estudio Nacional de Salud Bucal - ENSAB IV. Situación en Salud Bucal [Citado 14 agosto 2017]. Colombia: MSPS; 2014. Disponible en: https://www.minsalud.gov.co/sites/r id/Lists/BibliotecaDigital/RIDE/VS/P $\mathrm{P} /$ ENSAB-IV-Situacion-Bucal-

Actual.pdf

4.- Vásquez N, Gutiérrez A. Prevalencia de Hendidura Labial y/o Palatina en Pacientes de dos Centros Hospitalarios del Estado Carabobo. [Internet] ODOUS CIENTIFICA. 2005[Citado 14 agosto 2017]; 6(1). Disponible en: http://servicio.bc.uc.edu.ve/odontolo gia/revista/v6n1/6-1-2.pdf

5.- Charry I, Aguirre M. Caracterización de los pacientes con labio y paladar hendido y de la atención brindada en el Hospital Infantil Universitario de Manizales. Archivos de Medicina. (Internet) 2010(citado 29 febrero 2017); 12(2):190-197. Disponible en: http://dspace.udla.edu.ec/bitstream/ 330/5071/1/UDLA-EC-TOD-201623.pdf

6.- España G. Caracterización de pacientes con labio y paladar hendido. [Internet]. Guatemala: Universidad Rafael Landívar; 2015 [Citado 08 septiembre 2017]. Disponible en: http://recursosbiblio.url.edu.gt/tesisj cem/2015/09/03/Espana-Lilly.pdf

7.- Periche F. Frecuencia de neonatos con fisura del paladar y labio leporino en dos Hospitales Minsa de la Región Lambayeque, periodo 2012- 2014. 
[Internet]. Perú: Universidad Católica Santo Toribio De Mogrovejo; [Citado 08 septiembre 2017]. Disponible en: http://tesis.usat.edu.pe/bitstream/us at/327/1/TL_Periche_Fiestas_Cecilia. pdf

8.- Barrios Z, Salas M. Características epidemiológicas en pacientes pediátricos con hendiduras de labio y paladar. [Internet]. Venezuela: Universidad de Los Andes Medula; 2009. [Citado 28 de enero 2017]. Disponible en: http://www.saber.ula.ve/bitstream/1 23456789/42324/1/art7.pd

9.- Hernández M, Guerra M. Prevalencia de Hendiduras de labio y/o palatinas en los pacientes que acudieron al Centro de Investigación y Atención a Pacientes con Malformaciones Cráneo-faciales y Prótesis Maxilofacial. 2000-2012. [Internet] Acta Odontológica de Venezuela. 2013[Citado 28 de enero 2017]; 51(3) Disponible en: https://www.actaodontologica.com/e diciones/2013/3/art-11/

10.- Coiffman, F. Cirugía plástica, reconstructiva y estética. Barcelona España: SALVAT; 1996

11.- Rossell, P. Nueva clasificación de severidad de Fisuras Labiopalatinas del Programa Outreach Surgical Center Lima - Perú. Acta Méd. Peruana.2006 (Consultado 23 febrero 2017); 23(2):59-66

12.- Rossell $\mathrm{P}$, Cotrina O. Técnica de un colgajo: una nueva alternativa en el tratamiento de las fisuras palatinas. 2014 (Internet) Acta Médica Peruana. 31(3): 181-186. [Citado 04 enero 2017]. Disponible en: http://www.redalyc.org/articulo.oa?i $d=96634183007$

13.- Ford A. Tratamiento actual de las fisuras labiopalatinas. 2014(Internet) Med. Clin. Condes 15(1): 3-4. Disponible

en:http://www.clc.cl/clcprod/media/ contenidos/pdf/MED_15_4/Tratamie ntoLabiopalatinas

14.- Sadler, TW. Langman Embriología Médica. Madrid: Lippincontt Williams; 2008
15.- Moore, K. Embriología clínica. Rio de Janeiro: Elsevier; 2008

16.- Contreras F. Incidencia de labio y paladar hendido en el Hospital General "Dr. Aurelio Valdivieso" del estado de Oaxaca de 2008 a 2010. Cirugía y Cirujanos. [Internet] 2012 [Citado 04 enero 2017]; 80(4):339344. Disponible en: http://www.redalyc.org/articulo.oa?i $\mathrm{d}=66224459006$.

17.- Sorolla J. Anomalías Cráneo-faciales. Rev. Med. Clin. Condes. [Internet]; 2010(citado 13 marzo 2017); 21(1):515.

Disponible en:http://www.clinicalascondes.cl/De v_CLC/media/Imagenes/PDF\%20revi sta\%20m\%C3\%A9dica/2010/1\%20e nero/001

18.- Jalili D, Fathi M, Jalili C. Frequency of Cleft Lip and Palate among Live Births in Akbar Abadi Hospital. Acta Médica Iranica. [Internet] 2012[Consultado 06 enero 2017]; 50 (10):704-706. Disponible en: http://www.ncbi.nlm.nih.gov/pubme d/232752

19.- Fonseca R, Marciani R, Turvey T. Oral and Maxillofacial Surgery: Orthognathic surgery, esthetic surgery, cleft and craniofacial surgery $2^{\text {nd }}$ edition. Saunders/Elsevier. Philadelphia 2009, pp: 713-734

20.- Mena-Olalde J, González-Díaz I, Venegas-Gómez T, González-Díaz V, Medina-Aguilar S. Epidemiologia descriptiva de hendiduras labiopalatinas en la Clínica de Labio y Paladar Hendidos de Morelia, Michoacán, México (1989-2012) y su comparación con algunas poblaciones internacionales. Cir. Plást. Iberolatinoam. [Internet]. $2017 \mathrm{Mar}$ [citado 2020 Mar 15]; 43(1): 41-45. Disponible en: http://scielo.isciii.es/scielo.php?script =sci_arttext \&pid=S037678922017000 100006\&lng=es

21.- Cherfên B, Gómez C, Abreu A, et al.2009. Cuidados bucales en pacientes con fisuras de labio y/o paladar. Acta Odontológica Venezolana; 47:1-9. Disponible en: 
http://saber.ucv.ve/ojs/index.php/re v_aov/article/view/231/200

22.- Quijano M, Rivas J, Salas I, et al.2009. Aspectos sociodemográficos y clínicos del labio leporino y paladar fisurado en una población del suroccidente colombiano. Rev Fac Ciencias de la Salud. Universidad del Cauca. Colombia; 11: 25-30. Disponible en: http://www.facultadsalud.unicauca.e du.co/Revista\%5CPDF\%5C2009\%5C1 10103200903.pdf

23.- Chavarriaga J, González M. 2010. Prevalencia de labio y paladar hendido: Aspectos generales que se deben conocer. Rev Nal de Odontología; 6: 70-81. Disponible en: http://wb.ucc.edu.co/revistanacional deodontologia/files/2011/09/articul o-09-vol6n11.pdf

24.- Souza J, Raskin S. Clinical and epidemiological study of orofacial clefts. Revista de Pediatría. Rio de Janeiro-Brasil. [Internet]
2013[Citado02 marzo 2017]; 89(2):137-144. Disponible en: http://www.scielo.br/pdf/jped/v89n 2/en_v89n2a06.pd

25.- Mejía A, Suárez D. Factores de riesgo materno predominantes asociados con labio leporino y paladar hendido en los recién nacidos. Arch Inv Mat Inf. México. [Internet] 2012[Citado 04 enero 2017]; 4(2):55-62. Disponible en:

http://www.medigraphic.com/mater noinfantil

26.- Bedón M, Villota L. Labio y Paladar Hendido: Tendencias actuales en el manejo exitoso. Revisión de tema. Archivos de Medicina. (Internet) 2012[Citado 23 febrero 2017]; 12(1):107-119.

27. - Mossey PA., Little J, Munger RG, Dixon MJ, y Shaw WC. Cleft lip and palate. The Lancet, (2009) 374 :( 9703), 1773-1785 